\title{
Hospital water filters as a source of Mycobacterium avium complex
}

\author{
Correspondence \\ Joseph O. Falkinham, III \\ jofiii@vt.edu
}

Received 17 May 2010

Accepted 26 June 2010

\author{
Joseph O. Falkinham, III
}

Department of Biological Sciences, Virginia Polytechnic Institute and State University, Blacksburg, VA 24061-0406, USA

\begin{abstract}
Bronchoscopes and the filters used for washing them were found to yield high numbers of Mycobacterium avium isolates sharing the same repetitive sequence-based PCR (rep-PCR) fingerprint pattern as $M$. avium isolates recovered from patient samples collected by bronchoscopy. Water and biofilm samples collected from the bronchoscopy preparation laboratory yielded M. avium, Mycobacterium intracellulare, Mycobacterium malmoense and Mycobacterium gordonae. Several $M$. avium and $M$. intracellulare isolates from water samples in the bronchoscopy laboratory had rep-PCR patterns matching those of patient bronchoscopy isolates. Five of the 22 (23\%) M. avium patient bronchoscopy isolates and 42 of the 56 (75\%) M. intracellulare patient bronchoscopy isolates could have been due to contamination from the water supply.
\end{abstract}

\section{INTRODUCTION}

Reports of the isolation of non-tuberculous mycobacteria (NTM) from contaminated bronchoscopes appear regularly in the literature. Such investigations are usually prompted by the isolation of NTM from patient samples collected by bronchoscopy that are inconsistent with patient symptoms or conditions. In a number of instances, either inadequate bronchoscope disinfection (Rodrigues et al., 2001; Wang et al., 1995; Wheeler et al., 1989) or contamination by a bronchoscope-washing machine (Brown et al., 1993; Gillespie et al., 2000; Gubler et al., 1992; Kressel \& Kidd, 2001; Takigawa et al., 1995) was identified as the reason for the presence of NTM in the bronchoscopes. In addition, water used for washing bronchoscopes has been shown to be the source of NTM contaminating bronchoscopes (Bennett et al., 1994; Nye et al., 1990; Stine et al., 1987; Vijayaraghavan et al., 2006). In spite of these studies, there are only two reports where a DNA fingerprinting technique was employed to prove that the water used for washing the bronchoscopes was the source (Bennett et al., 1994; Chroneou et al., 2008).

The presence of NTM in the water supplies of bronchoscope-processing laboratories is consistent with their presence in hospital water (du Moulin et al., 1988) and municipal drinking water (Falkinham et al., 2001). However, source identification is not straightforward, as the wide genetic diversity within NTM species requires typing of patient and environmental isolates. In addition, in the absence of NTM colony counts, it is impossible to identify which source might be of primary importance and require remediation.

Abbreviations: NTM, non-tuberculous mycobacteria; rep-PCR, repetitive sequence-based PCR.
At a large general community hospital, Mycobacterium avium or Mycobacterium intracellulare isolates were recovered from 75 patient bronchoscopy samples collected over the period December 2007-February 2009, whose appearance was inconsistent with patient symptoms or conditions. The objective of our study was to collect samples from the hospital water system and bronchoscopyprocessing laboratory, test them for the presence of mycobacteria, and, if recovered, compare the DNA fingerprints of the patient and environmental isolates.

\section{METHODS}

Patient (bronchoscopy) NTM isolates. Twenty-two M. avium and $56 M$. intracellulare isolates cultured from patient bronchoscopy samples were obtained from the Maryland State Health Department Tuberculosis Laboratory.

Samples and NTM isolation, enumeration and identification. A total of 39 water and biofilm (swab) samples were collected from the bronchoscopy-processing laboratory (Table 1) by hospital staff and processed for the isolation, enumeration and identification of isolates as described by Falkinham et al. (2001). Briefly, $0.1 \mathrm{ml}$ water samples were spread directly in triplicate on Middlebrook 7H10 agar medium $(\mathrm{M} 7 \mathrm{H} 10)$ and the number of c.f.u. ( $\mathrm{ml} \mathrm{sample})^{-1}$ was reported (Table 1). Swabs used for the collection of surface biofilms were placed in $2 \mathrm{ml}$ sterile tap water and vortexed, and $0.1 \mathrm{ml}$ suspension was spread in triplicate on M7H10 agar and the results reported as c.f.u. per swab (Table 1). Six individual filters, all part of the same unit, were retrieved from the filter housings, placed in sterile bags and shipped to the Virginia Polytechnic Institute and State University laboratory for processing. For the filters, $1 \mathrm{~cm}^{2}$ of the surface of both the inlet and outlet sides of the filters was swabbed and the swabs were placed in $2 \mathrm{ml}$ sterile tap water and vortexed; $0.1 \mathrm{ml}$ of the suspension was spread on $\mathrm{M} 7 \mathrm{H} 10$ agar medium in triplicate and the results reported as c.f.u. $\mathrm{cm}^{-2}$. All samples were spread directly on M7H10 
Table 1. Hospital samples and mycobacterial recovery

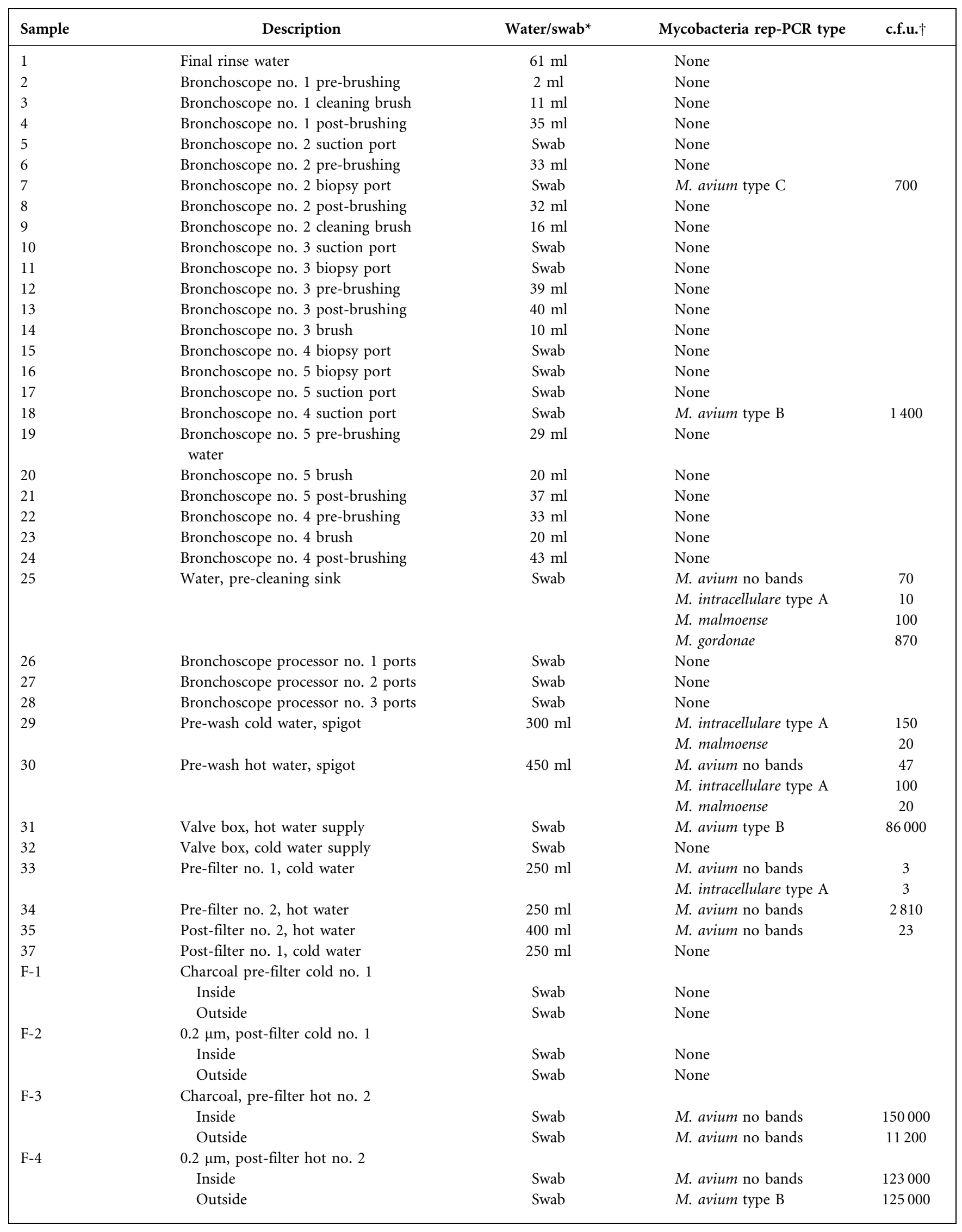


Table 1. cont.

\begin{tabular}{|c|c|c|c|c|}
\hline Sample & Description & Water/swab ${ }^{\star}$ & Mycobacteria rep-PCR type & c.f.u. $\dagger$ \\
\hline \multirow[t]{3}{*}{ F-5 } & Endoscopy pre-filter & & & \\
\hline & Inside & Swab & M. avium type B & 60 \\
\hline & Outside & Swab & M. avium type B & 3040 \\
\hline \multirow[t]{3}{*}{ F-6 } & Endoscopy filter & & & \\
\hline & Inside & Swab & M. avium type B & 20000 \\
\hline & Outside & Swab & M. avium type $\mathrm{B}$ & 90 \\
\hline \multicolumn{5}{|c|}{$\begin{array}{l}\text { Post-remediation } \\
\text { measures }\end{array}$} \\
\hline 38 & Endoscope water, post filter and UV & $900 \mathrm{ml}$ & None & \\
\hline 39 & Endoscope water, post filter & $900 \mathrm{ml}$ & None & \\
\hline
\end{tabular}

${ }^{*}$ Volume of water sample collected or volume of water in tube with bronchoscope brush. Swabs were placed in $2 \mathrm{ml}$ sterile tap water and vortexed for $60 \mathrm{~s}$ at maximum speed.

$\dagger$ Results for water samples expressed as c.f.u. $(\mathrm{ml} \text { sample) })^{-1}$, as c.f.u. per swab for swab samples and as c.f.u. $\mathrm{cm}^{-2}$ for filter samples.

agar, as decontamination was not necessary. Representative colonies of each putative NTM type were counted and streaked for isolation, and acid-fast isolates were identified by nested PCR of the 16S rRNA gene (Wilton \& Cousins, 1992) and the size of restriction fragments of the hsp-65 gene amplified by PCR (Telenti et al., 1993). Repetitive sequence-based PCR (rep-PCR) was employed for DNA fingerprint typing (Cangelosi et al., 2004) using DNA isolated using an UltraClean Microbial DNA Isolation kit (MoBio Laboratories) following the manufacturer's instructions. For identification and typing, reactions were performed twice using two separate DNA samples for each isolate.

\section{RESULTS}

\section{Recovery of NTM from samples}

Mycobacteria, comprising M. avium, M. intracellulare, Mycobacterium malmoense and Mycobacterium gordonae, were recovered from 17 of the 51 hospital samples $(33 \%)$ (Table 1). Extremely high numbers of M. avium $(\sim 10000$ $150000 \mathrm{~cm}^{-2}$ ) were recovered from the inside and outside of the two filters in the hot water line used for washing bronchoscopes (Table 1). Considering the surface area of the filters (i.e. $50 \times 6 \mathrm{~cm}$ diameter, and with a central $1 \mathrm{~cm}$ diameter hole), they harboured between 12 million and 188 million $M$. avium cells on the surface alone. $M$. avium was also recovered from two bronchoscope samples (Table 1).

\section{Fingerprint comparisons}

All M. avium and $M$. intracellulare isolates were fingerprinted by rep-PCR and unique band patterns were identified by letter (Fig. 1). Four unique band patterns ( $\mathrm{B}, \mathrm{C}, \mathrm{D}$ and $\mathrm{E}$ ) were displayed by the patient $\mathrm{M}$. avium bronchoscopy isolates and six (A, F, G, H, I and J) by the M. intracellulare isolates (Fig. 1 and data not shown). Hospital sample M. avium isolates belonged to types B and $\mathrm{C}$ and M. intracellulare isolates belonged to type A (Table 1). Five of the $22(23 \%)$ M. avium patient bronchoscopy isolates belonged to type B and 42 of the $56(75 \%) M$. intracellulare patient bronchoscopy isolates exhibited a type A pattern.

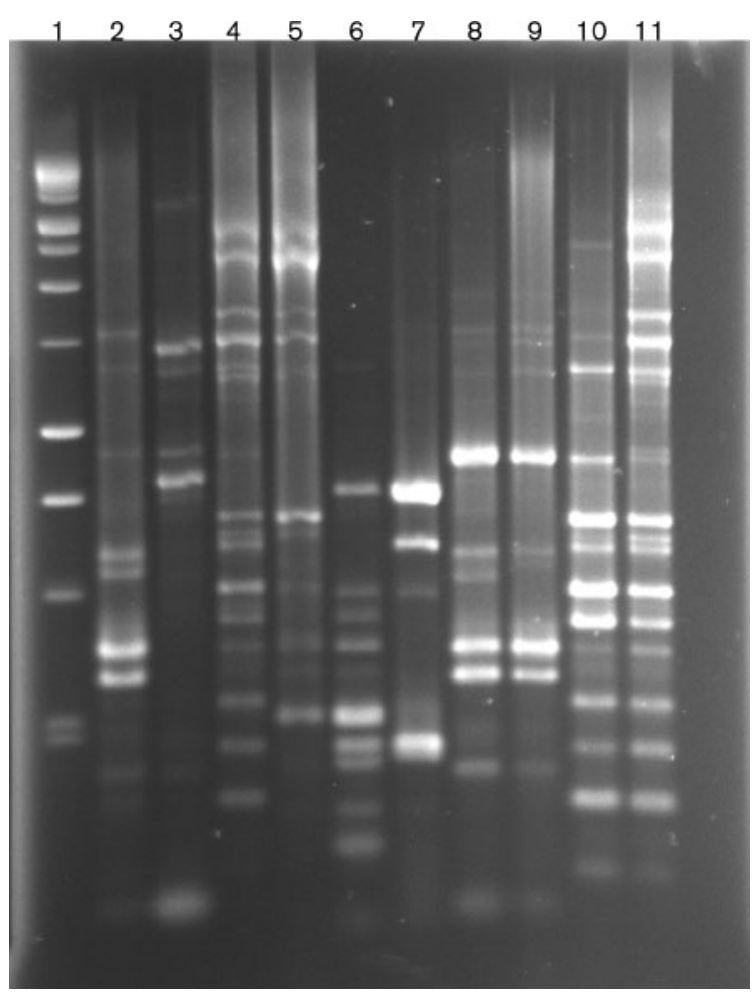

Fig. 1. Rep-PCR patterns of patient (lanes 2-7, rep-PCR types $B$, $C, A, F, G$ and $H$, respectively) and bronchoscope preparation laboratory (lanes $8-11$, rep-PCR types $B$ and $C$ ) isolates. Lanes: 1, $1 \mathrm{~kb}$ ladder; $2, M$. avium type $B ; 3, M$. avium type $C ; 4, M$. intracellulare type $A ; 5, M$. intracellulare type $F ; 6, M$. intracellulare type $\mathrm{G} ; 7, M$. intracellulare type $\mathrm{H} ; 8, M$. avium isolate $\mathrm{Sw}-7-1$ type $B ; 9, M$. avium isolate $W-18-1$ type $B ; 10, M$. intracellulare isolate W-29-1 type $C ; 11, M$. intracellulare isolate $W-30-2$ type $C$. 


\section{DISCUSSION}

The data demonstrated that isolation of mycobacteria from bronchoscopy samples was due to contamination of bronchoscopes during their preparation for use, based on the presence of the $M$. intracellulare rep-PCR type A and $M$. avium type B in filters and water in the bronchoscopy preparation laboratory. It is likely that the source of $M$. intracellulare rep-PCR type A contamination was the cold (sample 29) and hot (sample 30) water and that for $M$. avium rep-PCR type B was filter F-4 on the hot water line (Table 1). The endoscope filters also yielded M. avium repPCR type B (Table 1). The presence of M. avium and $M$. intracellulare in hot water is consistent with the heat resistance of the $M$. avium complex: more than $10 \%$ survive 3-4 h exposure to $60{ }^{\circ} \mathrm{C}$ (Schulze-Röbbecke \& Buchholtz, 1992).

Remediation measures included a review of the procedures for meticulous cleaning of bronchoscopes and installation of new filters in the existing filtration equipment and a UV sterilization device. The existing non-microbiological, inline cartridge-type water filters had been in place for more than 6 months. Point-of-use filters $(0.1 \mu \mathrm{m}$ pore size $)$ that prevent the passage of micro-organisms were installed on all taps providing water or flushing bronchoscopes. It is planned that the filters will be changed every 3 weeks following the manufacturer's directions. Furthermore, bronchoscopes are now air dried vertically to avoid storage of bronchoscopes containing liquid. It is anticipated that these measures will prevent the filters becoming the NTM source (Hilborn et al., 2006; Rodgers et al., 1999). The numbers of $M$. avium, $M$. intracellulare, $M$. malmoense and M. gordonae were reduced to below detectable levels (i.e. $<10$ c.f.u. $\mathrm{ml}^{-1}$ ) following the remediation measures (Table 1). The Centers for Disease Control and Prevention Guidelines for Environmental Infection Control in Health-care Facilities (http://www.cdc.gov/mmwr/preview/mmwrhtml/ rr5210a1.htm) includes guidelines for infection control of bronchoscopes.

A second noteworthy observation from this study was the very high numbers of $M$. avium in filters. This is believed to be the first report demonstrating that filters are a source of M. avium contaminating bronchoscopes. Although filters can be effective in reducing the numbers of $M$. avium in water lines, they can become sources of $M$. avium, presumably due to saturation and growth followed by release (Hilborn et al., 2006; Rodgers et al., 1999; Stine et al., 1987). The water filters employed in the hospital described here were not microbiological filters, i.e. the pore size did not prevent the passage of NTM and other bacterial cells. The filters simply reduced the number of NTM passing through because of the longer pathway required for passage. However, as the filters trap nutrients, NTM can grow and eventually they pass through the filter. To be effective, the filters must be replaced at 3-week intervals to allow for continual removal of NTM from water (Rodgers et al., 1999). Furthermore, high numbers of
NTM in the filters and consequently in the bronchoscopes could also have reduced disinfectant efficiency, as has been shown with M. gordonae (Jackson et al., 1996).

\section{ACKNOWLEDGEMENTS}

The author wishes to acknowledge the Maryland State Health Department Tuberculosis Laboratory for providing the patient NTM isolates and the technical assistance of Ms Myra D. Williams who performed the isolation, enumeration and identification of NTM from the samples submitted by the hospital. Financial support for this work was provided by funds from Applied Microbiology and Genetics through the Virginia Tech Foundation.

\section{REFERENCES}

Bennett, S. N., Peterson, D. E., Johnson, D. R., Hall, W. H., RobinsonDunn, B. \& Dietrich, S. (1994). Bronchoscopy-associated Mycobacterium xenopi pseudoinfections. Am J Respir Crit Care Med 150, 245-250.

Brown, N. M., Hellyar, E. A., Harvey, J. E. \& Reeves, D. S. (1993). Mycobacterial contamination of fibreoptic bronchoscopes. Thorax 48 , 1283-1285.

Cangelosi, G. A., Freeman, R. J., Lewis, K. N., Livingston-Rosanoff, D., Shah, K. S., Milan, K. J. \& Goldberg, S. V. (2004). Evaluation of a highthroughput repetitive-sequence-based PCR system for DNA fingerprinting of Mycobacterium tuberculosis and Mycobacterium avium complex strains. J Clin Microbiol 42, 2685-2693.

Chroneou, A., Zimmerman, S. K., Cook, S., Willey, S., Eyre-Kelly, J., Zias, N., Shapiro, D. S., Beamis, J. F. \& Craven, D. E. (2008). Molecular typing of Mycobacterium chelonae isolates from a pseudooutbreak involving an automated bronchoscope washer. Infect Control Hosp Epidemiol 29, 1088-1090.

du Moulin, G. C., Stottmeier, K. D., Pelletier, P. A., Tsang, A. Y. \& Hedley-Whyte, J. (1988). Concentration of Mycobacterium avium by hospital hot water systems. JAMA 260, 1599-1601.

Falkinham, J. O., III, Norton, C. D. \& LeChevallier, M. W. (2001). Factors influencing numbers of Mycobacterium avium, Mycobacterium intracellulare, and other mycobacteria in drinking water distribution systems. Appl Environ Microbiol 67, 1225-1231.

Gillespie, T. G., Hogg, L., Budge, E., Duncan, A. \& Coia, J. E. (2000). Mycobacterium chelonae isolated from rinse water within an endoscope washer-disinfector. J Hosp Infect 45, 332-334.

Gubler, J. G., Salfinger, M. \& von Graevenitz, A. (1992). Pseudoepidemic of nontuberculous mycobacteria due to a contaminated bronchoscope cleaning machine. Chest 101, 1245-1249.

Hilborn, E. D., Covert, T. C., Yakrus, M. A., Harris, S. I., Donnelly, S. F., Rice, E. W., Toney, S., Bailey, S. A. \& Stelma, G. N., Jr (2006). Persistence of nontuberculous mycobacteria in a drinking water system after addition of filtration treatment. Appl Environ Microbiol 72, 5864-5869.

Jackson, J., Leggett, J. E., Wilson, D. \& Gilbert, D. N. (1996). Mycobacterium gordonae in fiberoptic bronchoscopes. Am J Infect Control 24, 19-23.

Kressel, A. B. \& Kidd, R. (2001). Pseudo-outbreak of Mycobacterium chelonae and Methylobacterium mesophilicum caused by contamination of an automated endoscopy washer. Infect Control Hosp Epidemiol 22, 414-418.

Nye, K., Chadha, D. K., Hodgkin, P., Bradley, C., Hancox, J. \& Wise, R. (1990). Mycobacterium chelonei isolation from broncho-alveloar lavage fluid and its practical applications. J Hosp Infect 16, 257-261. 
Rodgers, M. R., Blackstone, B. J., Reyes, A. L. \& Covert, T. C. (1999). Colonisation of point of use water filters by silver resistant nontuberculous mycobacteria. J Clin Pathol 52, 629.

Rodrigues, C., Mehta, A., Jha, U., Bharucha, M., Dastur, F. D. \& Udwadia, T. E. (2001). Nosocomial Mycobacterium chelonac infection in laparoscopic surgery. Infect Control Hosp Epidemiol 22, 474-475.

Schulze-Röbbecke, R. \& Buchholtz, K. (1992). Heat susceptibility of aquatic mycobacteria. Appl Environ Microbiol 58, 1869-1873.

Stine, T. M., Harris, A. A., Levin, S., Rivera, N. \& Kaplan, R. L. (1987). A pseudoepidemic due to atypical mycobacteria in a hospital water supply. JAMA 258, 809-811.

Takigawa, K., Fujita, J., Negayama, K., Terada, S., Yamaji, Y., Kawanishi, K. \& Takahara, J. (1995). Eradication of contaminating Mycobacterium chelonae from bronchofibrescopes and an automated bronchoscope disinfection machine. Respir Med 89, 423-427.

Telenti, A., Marchesi, F., Balz, M., Bally, F., Böttger, E. C. \& Bodmer, T. (1993). Rapid identification of mycobacteria to the species level by polymerase chain reaction and restriction enzyme analysis. J Clin Microbiol 31, 175-178.

Vijayaraghavan, R., Chandrashekhar, R., Sujatha, Y. \& Belagavi, C. S. (2006). Hospital outbreak of atypical mycobacterial infection of port sites after laproscopic surgery. J Hosp Infect 64, 344347.

Wang, H.-C., Liaw, P.-C., Yang, S.-H. \& Luh, K. T. (1995). A pseudoepidemic of Mycobacterium chelonae infection caused by contamination of a fiberoptic bronchoscope suction channel. Eur Respir J 8, 1259-1262.

Wheeler, P. W., Lancaster, D. \& Kaiser, A. B. (1989). Bronchopulmonary cross-contamination and infection related to mycobacterial contamination of suction valves of bronchoscopes. J Infect Dis 159, 954-958.

Wilton, S. \& Cousins, D. (1992). Detection and identification of multiple mycobacterial pathogens by DNA amplification in a single tube. PCR Methods Appl 1, 269-273. 